de Catalunya. 468 pp. Barcelona.

VILLAR, L. \& J.L. BENITO ALONSO -1995-

Esquema de la vegetación del Parque Nacional de Ordesa y Monte Perdido, más su zona periférica. Lucas Mallada 6: 235-273.

VILLAR, L., J.A. SESÉ \& J.V. FERRÁNDEZ 1997- Atlas de la Flora del Pirineo Aragonés, I (Introducción. Lycopodiaceae-Umbelliferae). Consejo de Protección de la Naturaleza de Aragón e Instituto de Estudios Altoaragoneses. XCI+648 pp. Huesca.
VILLAR, L., J.A. SESÉ \& J.V. FERRÁNDEZ -en prensa- Atlas de la Flora del Pirineo Aragonés, II (Ericaceae-Orchidaceae). Consejo de Protección de la Naturaleza de Aragón e Instituto de Estudios Altoaragoneses. Huesca.

Aceptado para su publicación en julio de 2000

Dirección del autor. Instituto Pirenaico de Ecología, C.S.I.C. Apdo. 64. E-22700 Jaca. Huesca. España. C. e.: jlbenito@aragob.es

\title{
94. CONTRIBUCIONES AL CONOCIMIENTO DE LA ALIANZA DESCHAMPSION MEDIAE BR.-BL. IN BR.-BL.ET AL. 1952 EN LAS SIERRAS SUBBÉTICAS DEL SUR DE LA PENÍNSULA IBÉRICA
}

\author{
Juan Antonio TORRES, Antonio GARCÍA-FUENTES, \\ Carlos SALAZAR, Manuel MELENDO y Eusebio CANO
}

Contributions to the knowledge of the Deschampsion mediae Br.-Bl. in Br.-Bl. et al. 1952 alliance in the Subbetic Mountains of the southern Iberian Peninsula.

Palabras clave. Deschampsion mediae, sierras Subbéticas, Andalucía, España.

Key words. Deschampsion mediae, Subbetic Mountains, Andalusia, Spain.

Las características ecológicas de los ambientes donde existen comunidades de la clase Molinio-Arrhenatheretea Tüxen 1937, suelos profundos más o menos húmedos, permiten una gran diversidad florística y de comunidades vegetales. Así, dentro de toda la gama de herbazales y juncales que prosperan en el seno de la clase aparecen diferenciados diversos ordenes (Rivas Martínez et al., 1999) en función de su distribución, grado de hidromorfía e influencia antropozoógena. De entre los órdenes de óptimo mediterráneo, Holoschoenetalia Br.-B1. ex Tchou 1948 incluye dos alianzas: Molinio-Holoschoenion Br.-Bl. ex Tchou 1948 dominada por juncales de Scirpus holoschoenus indiferentes a la naturaleza química del sustrato, y Deschampsion mediae $\mathrm{Br}$--Bl. in $\mathrm{Br}$ - $\mathrm{Bl}$. et al. 1952, representada por pastizales agostantes con dominio de biotipos hemicriptofítico y camefítico, desarrollados sobre sustratos carbonatados, especialmente margas ricas en 
arcillas, que originan suelos impermeables, húmedos o ligeramente encharcados en invierno y primavera, pero que sufren una acusada desecación estival, y con una distribución mediterráneo-iberolevantina y bética oriental, con óptimo en bioclimas subhúmedo-húmedos meso-supramediteráneos.

En este trabajo se estudian estos pastizales de la alianza Deschampsion mediae Br.-Bl. in Br.-Bl. et al. 1952, aportando datos sobre su estado actual en la península Ibérica, e incidiendo sobre su presencia en las sierras Subbéticas del sur peninsular, donde se propone una nueva asociación, Eryngio dilatatiJasonietum tuberosae Torres \& Cano ass. nov., y se comentan aspectos sobre su estructura, factores ecológicos, corológicos y sintaxonómicos.

Para la flora comentada en el texto se siguen por orden preferencial las obras de Castroviejo et al. (1986-1999), Tutin et al. (1964-1980) y Valdés et al. (1987), a excepción de Deschampsia hispanica (Vivant) Cervi \& Romo in Collect. Bot. 12:82. 1981. La tipología bioclimática y fitogeográfica está basada en Rivas Martínez (1996) y Rivas Martínez et al. (1997), respectivamente.

En el esquema sintaxonómico actual de la alianza Deschampsion mediae en la Península Ibérica (tab. 1) es posible diferenciar dos grupos de comunidades vegetales caracterizadas por uno u otro cortejo florístico en función de la duración e intensidad del encharcamiento. En un primer grupo, Deschampsia média y Deschampsia hispanica constituyen el nucleo florístico de la comunidad (Deschampsietum mediae Br.-Bl. in Br.-B1., Roussine \& Nègre 1952, Sanguisorbo laterifloraeDeschampsietum hispanicae Rivas Martínez \& G. López in G. López 1978 corr. Rivas Martínez, Fernández González, Sánchez Mata \& Pizarro 1990), generalmente con aspecto de praderas densas, ricas en hemicriptófitos graminoides que permanecen húmedas o con aguas remansadas durante la mayor parte del año, y que al llegar el periodo estival sufren fuerte desecación con aparición de grandes grietas de retracción. En este tipo de comunidades es muy frecuente la formación de macollas de superficie y extensión irregular sobre los sustratos blandos de naturaleza margosa.

En un segundo grupo, se incluyen una serie de comunidades de ambientes más secos (Plantagini serpentinae-Jasonietum tuberosae O. Bolòs \& Masalles in O. Bolòs 1983, Agrostio stoloniferae-Achilleetum agerati $\mathrm{Br}$.-Bl. in $\mathrm{Br}$.B1., Roussine \& Nègre 1952, Prunello hyssopifoliae-Plantaginetum serpentinae Biurrun 1999), con suelos normalmente más compactos, a veces más guijarrosos e incluso pedregosos, donde los niveles de hidromorfía suelen ser inferiores, preferentemente en la estación primaveral y con una rápida desecación al comienzo del estío. En estos casos suelen dominar especies perennes rizomatosas bioindicadoras de menor humedad, sobre todo de las familias compuestas y labiadas, mientras que el género Deschampsia no suele aparecer, o en su caso, juega un papel secundario en la asociación vegetal sin llegar a formar tapices densos.

La asociación Deschampsietum mediae fue descrita para toda la llanura del Languedoc francés, Provenza occidental y, de forma fragmentaria, en el bajo Cévennes. En la Península Ibérica es frecuente en el prepirineo aragonés y catalán (Bolòs, 1959, 1967, 1983; Romo, 1989) alcanzando su área marginal en los montes de Prades catalanes, donde ya aparece de forma fragmentaria. Hacia el interior de la península alcanza el Sistema Ibérico en las sierras de Gúdar y Javalambre (Rivas Goday \& Borja, 1961). En su composición florística destacan Deschampsia media, Thymelaea passerina y Seseli elatum junto a Prunella hyssopifolia, Plantago maritima subsp. serpentina, Centaurea jacea, Agrostis stolonifera y Lotus tenuis (tab. 1).

Las praderas de Sanguisorbo lateriflorae- 
Deschampsietum hispanicae sustituyen al Deschampsietum mediae en el interior de la Península y en su composición florística destacan Deschampsia hispanica, Sanguisorba lateriflora, Jasonia tuberosa, Achillea ageratum y Leontodon carpetanus. Descrita por sus autores para la serranía de Cuenca podría extenderse a toda la provincia Castellano-Maestrazgo-Manchega, con penetraciones en el sector Subbético (López Vélez, 1996) (tab. 1).

La asociación Agrostio stoloniferaeAchilleetum agerati también fue puesta de manifiesto en las margas terciarias de la llanura del Languedoc francés, extendiéndose desde el prepirineo ibérico hacia el interior de diversas montañas catalánidas (Bolòs, 1983, 1996). En su composición florística destacan Cichorium pumilum, Achillea ageratum, Agrostis stolonifera, Blackstonia perfoliata subsp. serotina y Juncus striatus (tab. 1).

Plantagini serpentinae-Jasonietum tuberosae es una comunidad rica en Jasonia tuberosa, que al ocupar suelos más secos que el Deschampsietum mediae suele empobrecerse en especies características de la alianza; junto a la especie directriz destacan Plantago maritima subsp. serpentina, sobre todo en las vaguadas, y algunas especies características de orden y clase como Trifolium repens, $T$. pratense, Cynodon dactylon y Agrostis stolonifera. Se extiende por toda la baja montaña catalana mediterránea (Bolòs, 1959, 1983, 1996; Vigo, 1996) (tab. 1).

Prunello hyssopifoliae-Plantaginetum serpentinae forma pastizales semiagostantes caracterizados por Jasonia tuberosa, Plantago maritima subsp. serpentina, Prunella hypssopifolia, Carex flacca y Deschampsia cespitosa acompañados de otras especies características de orden y clase como Briza media, Lotus corniculatus y Centaurea jacea. Denunciada por diversos autores (García Mijangos, 1997; Loidi et al., 1997, Biurrun, 1999), presenta una distribución castellano- cantábrica y navarro-alavesa (territorios subhúmedos y húmedos inferiores), mesomediterránea superior y supramediterránea y mesotemplada superior (tab. 1).

Deschampsion mediae en las sierras Subbéticas: estructura, composición y dinámica

Como consecuencia de las investigaciones sobre la flora y vegetación realizadas en la Sierra Sur de la provincia de Jaén, encrucijada entre los parques naturales de Sierra Mágina y las Subbéticas Cordobesas, presentamos los resultados obtenidos del estudio de los pastizales vivaces semiagostantes que colonizan los ambientes subhúmedo-humedos de las masas forestales aclaradas. Se trata de un territorio localizado en las sierras Subbéticas de la provincia de Jaén, perteneciente biogeográficamente al sector Subbético de la provincia corológica Bética, con dominancia de termotipos meso y supramediterráneo y ombroclima seco-subhúmedo. Son mayoritarios los sustratos carbonatados, calizas y calizo-dolomías, así como margas y margocalizas bastante deleznables en algunos casos (García Dueñas, 1986) sobre los que se desarrollan mayoritariamente suelos de los tipos cambisoles cálcicos, regosoles calcáreos y litosoles (Aguilar et al., 1987).

\section{Eryngio dilatati-Jasonietum tuberosae} Torres \& Cano ass.nov. (tab. 2; holotypus inv. 2)

Fitocenosis propia de suelos margosos muy ricos en arcillas que se desarrolla en pequeñas vaguadas donde se producen afloramientos de agua, que rápidamente son colonizadas por hierbas perennes de raices gruesas, como Jasonia tuberosa, Carduncellus cuatrecasasii y Eryngium dilatatum. Aparece al principio de la primavera con la floración de los hemicriptófitos graminoides y se prolonga 


\begin{tabular}{lllllllllllllll}
\hline $\mathrm{N}^{\mathrm{o}}$ de inventarios & 22 & 16 & 17 & 2 & 2 & 10 & 5 & 7 & 9 & 8 & 5 & 15 & 6 & 7 \\
Orden de inventarios & 1 & 2 & 3 & 4 & 5 & 6 & 7 & 8 & 9 & 10 & 11 & 12 & 13 & 14 \\
\hline
\end{tabular}

Características de asociación y alianza Prunella hyssopifolia

Jasonia tuberosa

Centaurea jacea

Plantago maritima subsp. serpentina

Centaurium pulchellum

Trifolium lappaceum

Hypericum tomentosum

Cichorium pumilum

Carex flacca

Briza media

Achillea ageratum

Festuca fenas

Prunella laciniata

Deschampsia media

Seseli elatum

Thymelaea passerina

Deschampsia hispanica

Sanguisorba lateriflora

Leontodon carpetanus

Achillea millefolium

Carex distans

Linum bienne

Lotus glaber

Deschampsia cespitosa

Centaurea paniculata subsp. leucophaea

Potentilla neumanniana

Prunella grandiflora subsp. pyrenaica

Blackstonia perfoliata subsp. serotina

Juncus striatus

Centaurium spicatum

Centaurea jacea subsp. vinyalsii

Eryngium dilatatum

Centaurea castellana

Carduncellus cuatrecasasii

Linum tenue

\begin{tabular}{|c|c|c|c|c|c|c|c|c|c|c|c|c|c|}
\hline V & V & V & - & - & III & III & III & II & V & - & IV & - & III \\
\hline I & V & - & - & III & III & II & II & IV & V & V & - & I & - \\
\hline IV & V & II & III & - & II & - & III & III & IV & II & I & - & - \\
\hline IV & III & - & III & V & IV & - & $\mathrm{V}$ & V & V & $\mathrm{V}$ & I & I & - \\
\hline II & III & III & - & III & I & II & II & - & II & I & IV & I & - \\
\hline III & I & - & - & - & + & II & II & - & I & - & IV & - & - \\
\hline I & - & I & - & - & - & - & - & - & I & - & I & - & - \\
\hline I & - & - & - & - & - & - & - & - & - & - & V & - & - \\
\hline - & - & - & V & III & II & - & - & IV & V & II & - & - & - \\
\hline - & - & - & III & - & II & II & IV & - & IV & - & - & I & - \\
\hline - & - & - & - & III & IV & V & V & - & - & - & IV & V & III \\
\hline- & - & - & - & V & - & - & - & - & II & - & - & - & - \\
\hline- & - & - & - & - & - & I & - & - & - & II & - & I & - \\
\hline V & V & V & - & - & - & - & - & - & - & - & - & - & - \\
\hline II & V & - & - & - & - & - & - & - & - & - & - & - & - \\
\hline I & I & I & - & - & - & - & - & - & - & - & - & - & - \\
\hline- & - & - & $\mathrm{V}$ & $\mathrm{V}$ & V & $\mathrm{V}$ & $\mathrm{V}$ & II & - & - & - & - & - \\
\hline - & - & - & - & - & III & II & III & - & - & - & - & - & - \\
\hline - & - & - & - & - & II & - & III & - & - & - & - & - & - \\
\hline - & - & - & - & - & - & I & - & - & - & - & - & - & - \\
\hline - & - & - & - & - & - & - & - & - & I & - & - & - & - \\
\hline - & - & - & - & - & - & - & - & - & II & - & - & - & - \\
\hline - & - & - & - & - & - & - & - & - & I & - & - & - & - \\
\hline - & - & - & - & - & - & - & - & - & III & & - & - & - \\
\hline - & - & - & - & - & - & - & - & - & -1 & II & - & - & - \\
\hline - & - & - & - & - & - & - & - & - & - & II & - & - & - \\
\hline - & - & - & - & - & - & - & - & - & - & I & - & - & - \\
\hline - & - & - & - & - & - & - & - & - & - & - & III & - & - \\
\hline - & - & - & - & - & - & - & - & - & - & - & II & - & - \\
\hline - & - & - & - & - & - & - & - & - & - & - & II & - & - \\
\hline - & - & - & - & - & - & - & - & - & - & - & - & I & - \\
\hline - & - & - & - & - & - & - & - & - & - & - & - & - & $\mathrm{V}$ \\
\hline - & - & - & - & - & - & - & - & - & - & - & - & - & II \\
\hline - & - & - & - & - & - & - & - & - & - & - & - & - & V \\
\hline - & - & - & - & - & - & - & - & - & - & - & - & - & IV \\
\hline
\end{tabular}

Características de otras Alianzas, Orden y Clase

Agrostis stolonifera

Lotus tenuis

Juncus articulatus

Scirpus holoschoenus

Molinia caerulea

Trifolium pratense

Blackstonia perfoliata

Leucanthemum vulgare

Prunella vulgaris

Cynodon dactylon

Plantago lanceolata

Potentilla reptans

Trifolium fragiferum

Juncus inflexus

Phleum pratense

Lotus corniculatus

$\begin{array}{llllllllllllll}\text { V } & \text { IV } & \text { II } & - & - & \text { II } & \text { II } & \text { IV } & - & \text { II } & \text { IV } & \text { V } & \text { V } & - \\ \text { IV } & \text { III } & \text { IV } & - & \text { V } & - & - & - & - & - & - & \text { IV } & - & - \\ \text { I } & \text { I } & \text { I } & \text { V } & - & \text { II } & \text { III } & - & - & - & \text { III } & \text { II } & - & - \\ - & \text { I } & \text { II } & - & - & - & - & \text { II } & - & \text { I } & \text { II } & \text { IV } & - & - \\ - & \text { I } & \text { I } & - & \text { III } & - & - & \text { III } & - & - & - & - & - & - \\ - & - & - & \text { III } & \text { V } & \text { II } & - & \text { V } & - & \text { II } & \text { IV } & - & \text { IV } & - \\ - & - & - & \text { V } & - & - & - & - & - & \text { III } & \text { II } & - & \text { I } & - \\ - & - & - & \text { III } & - & - & - & - & - & - & - & - & \text { I } & - \\ - & - & - & - & \text { V } & - & \text { I } & \text { IV } & \text { III } & - & \text { II } & \text { I } & \text { I } & - \\ - & - & - & - & - & - & - & \text { III } & - & - & \text { II } & \text { V } & \text { II } & - \\ - & - & - & - & - & - & \text { II } & - & \text { III } & \text { I } & \text { II } & \text { V } & \text { IV } & - \\ - & - & - & - & - & \text { II } & - & \text { II } & - & \text { I } & \text { II } & \text { III } & \text { III } & - \\ - & - & - & - & - & \text { II } & \text { III } & \text { III } & - & \text { II } & \text { II } & \text { III } & \text { III } & - \\ - & - & - & - & \text { V } & - & - & \text { III } & - & - & - & \text { II } & - & - \\ - & - & - & - & - & - & - & \text { III } & - & - & \text { II } & - & \text { I } & - \\ - & - & - & - & - & \text { IV } & \text { III } & \text { V } & \text { II } & \text { IV } & \text { II } & - & \text { II } & -\end{array}$




\begin{tabular}{|c|c|c|c|c|c|c|c|c|c|c|c|c|c|c|}
\hline $\begin{array}{l}\mathrm{N}^{\circ} \text { de inventarios } \\
\text { Orden de inventarios }\end{array}$ & $\begin{array}{l}22 \\
1\end{array}$ & $\begin{array}{l}16 \\
2\end{array}$ & $\begin{array}{l}17 \\
3\end{array}$ & $\begin{array}{l}2 \\
4\end{array}$ & $\begin{array}{l}2 \\
5\end{array}$ & $\begin{array}{l}10 \\
6\end{array}$ & $\begin{array}{l}5 \\
7\end{array}$ & $\begin{array}{l}7 \\
8\end{array}$ & $\begin{array}{l}9 \\
9\end{array}$ & $\begin{array}{l}8 \\
10\end{array}$ & $\begin{array}{l}5 \\
11\end{array}$ & $\begin{array}{l}15 \\
12\end{array}$ & $\begin{array}{l}6 \\
13\end{array}$ & $\begin{array}{l}7 \\
14\end{array}$ \\
\hline Cichorium intybus & - & - & - & - & - & III & - & II & - & - & II & - & I & - \\
\hline Phleum bertolonii & - & - & - & - & - & IV & II & - & - & II & - & - & I & IV \\
\hline Hypochoeris radicata & - & - & - & - & - & - & - & III & - & I & IV & - & II & - \\
\hline Oenanthe pimpinelloides & I & - & - & - & - & - & - & - & - & - & - & II & - & - \\
\hline Inula viscosa & I & I & - & - & - & - & - & - & - & - & - & III & - & - \\
\hline Gladiolus illyricus & - & I & - & - & - & - & - & - & - & - & - & I & - & - \\
\hline Leontodon maroccanus subsp. taraxacoides & I & - & - & III & - & - & - & - & - & - & - & - & - & \\
\hline Tetragonolobus maritimus & - & - & I & - & - & - & - & - & - & - & - & I & - & - \\
\hline Festuca arundinacea & - & - & - & III & - & - & - & - & - & - & - & - & - & - \\
\hline Linum catharticum & - & - & - & III & - & - & - & - & - & - & - & - & - & - \\
\hline Ranunculus bulbosus subsp aleae & - & - & - & III & - & - & - & - & - & - & - & - & - & - \\
\hline Schoenus nigricans & - & - & - & III & - & - & - & - & - & - & - & - & - & - \\
\hline Lotus corniculatus subsp. delortii & - & - & - & III & - & - & - & - & - & - & - & - & I & - \\
\hline Medicago lupulina & - & - & - & III & - & - & - & - & - & - & - & - & I & - \\
\hline Poa pratensis & - & - & - & III & III & - & - & - & - & - & - & - & I & - \\
\hline Succisa pratensis & - & - & - & - & III & - & - & - & - & - & - & - & - & - \\
\hline Ranunculus acris & - & - & - & - & III & - & - & - & - & - & - & - & - & - \\
\hline Lathyrus pratensis & - & - & - & - & III & - & - & - & - & - & - & - & - & - \\
\hline Mentha longifolia & - & - & - & - & III & - & - & - & - & - & - & - & - & - \\
\hline Oenanthe lachenalii & - & - & - & - & III & - & - & - & - & - & - & - & - & - \\
\hline Filipendula vulgaris & - & - & - & - & - & I & - & - & - & - & - & - & - & - \\
\hline Festuca rubra subsp. tricophylla & - & - & - & - & - & I & - & - & - & - & - & - & - & - \\
\hline Trifolium repens & - & - & - & - & - & - & II & I & - & II & - & - & - & - \\
\hline Oenanthe peucedanifolia & - & - & - & - & - & - & I & - & - & - & - & - & - & - \\
\hline Serratula nodicaulis & - & - & - & - & - & - & I & - & - & - & - & - & - & - \\
\hline Festuca gr. rubra & - & - & - & - & - & - & - & IV & - & - & - & - & - & - \\
\hline Taraxacum officinale & - & - & - & - & - & - & - & II & - & - & - & - & - & - \\
\hline Ranunculus bulbosus & - & - & - & - & - & - & - & IV & - & I & - & - & - & - \\
\hline Carex flaca subsp. serratula & - & - & - & - & - & - & - & III & - & - & - & - & - & - \\
\hline Poa compressa & - & - & - & - & - & - & - & - & - & I & II & - & - & - \\
\hline Cirsium monspessulanum & - & - & - & - & - & - & - & - & - & I & - & - & - & - \\
\hline Carduncellus monspeliensis & - & - & - & - & - & - & - & - & - & - & II & - & - & - \\
\hline Salvia pratensis & - & - & - & - & - & - & - & - & - & - & II & - & - & - \\
\hline Verbena officinalis & - & - & - & - & - & - & - & - & - & - & II & - & II & - \\
\hline Pulicaria dysenterica & - & - & - & - & - & - & - & - & - & - & II & I & I & - \\
\hline Plantago media & - & - & - & - & - & - & - & - & - & - & - & - & I & - \\
\hline Plantago major & - & - & - & - & - & - & - & - & - & - & - & - & I & - \\
\hline Odontites verna subsp. serotina & - & - & - & - & - & + & - & - & - & - & - & - & I & - \\
\hline Agrostis castellana var. mutica & - & - & - & - & - & - & - & - & - & - & - & - & - & III \\
\hline Lotus uliginosus & - & - & - & - & - & - & - & - & - & - & - & - & - & III \\
\hline Juncus fontanesii & - & - & - & - & - & - & - & - & - & - & - & - & - & II \\
\hline
\end{tabular}

Procedencia de los inventarios: 1. Deschampsietum mediae (Br.-Bl. et al. 1952: 138-139, grupo a). 2. Deschampsietum mediae inuletosum (Br.-Bl. et al. 1952: 138-139, grupo b). 3. Deschampsietum mediae (Br.-Bl. et al. 1952 : 138-139, grupo c). 4. Deschampsietum mediae hispanicetosum (Romo 1989: tabla 3, inv. 6 y 7). 5. Sanguisorbo lateriflorae-Deschampsietum hispanicae (Rivas Goday \& Borja 1961:240, cuadro 58). 6. Sanguisorbo lateriflorae-Deschampsietum hispanicae (López González 1978: 651, tabla 23). 7. Sanguisorbo lateriflorae-Deschampsietum hispanicae (López Vélez 1996: 431, tabla 65). 8. Sanguisorbo laterifloraeDeschampsietum hispanicae (Mateo 1983: 137, Cuadro 15). 9. Prunello hyssopifoliae-Plantaginetum serpentinae (Garcia Mijangos 1997: 299, tabla 34). 10. Prunello hyssopifoliae-Plantaginetum serpentinae (Biurrun 1999: 61, tabla 20). 11. Plantagini serpentinaeJasonietum tuberosae (tabla sintética: Bolòs 1996, tabla 6, 2 inv.; Vigo 1996, tabla 12, 1 inv.; Bolòs 1959: 148, 1 inv.). 12. AgrostioAchilleetum agerati (Br.-Bl. et al. 1952: 136). 13. Agrostio- Achilleetum agerati (tabla sintética: Bolòs 1996, tabla 7, 4 inv.; Bolòs 1983, tabla 49, 1 inv.). 14 Eryngio dilatati-Jasonietum tuberosae Torres \& Cano ass. nov. (tabla 2 de este trabajo).

Tabla 1. Tabla sintética de las comunidades de Deschampsion mediae Br.-Bl. in Br.-Bl. et al. 1952 en la península Ibérica. 
Tabla 2

Eryngio dilatati-Jasonietum tuberosae Torres \& Cano, ass. nov.

(Deschampsion mediae, Holoschoenetalia, Molinio-Arrhenatheretea)

\begin{tabular}{|c|c|c|c|c|c|c|c|c|c|c|}
\hline Área inventariada $\left(\mathrm{m}^{2}\right)$ & 15 & 20 & 20 & 20 & 20 & 10 & 20 & 10 & 20 & 15 \\
\hline Altura media (cms.) & 25 & 30 & 30 & 30 & 20 & 20 & 25 & 20 & 20 & 25 \\
\hline Cobertura (\%) & 70 & 80 & 80 & 80 & 80 & 95 & 40 & 80 & 80 & 80 \\
\hline Altitud $(1=10 \mathrm{~m})$ & 130 & 105 & 110 & 118 & 130 & 136 & 124 & 120 & 120 & 105 \\
\hline Inclinación (\%) & 3 & 3 & 5 & 3 & - & - & 3 & - & - & - \\
\hline Orientación & $\mathrm{NE}$ & $\mathrm{N}$ & $\mathrm{N}$ & $\mathrm{N}$ & - & - & W & - & - & - \\
\hline Num. Especies & 22 & 15 & 21 & 16 & 22 & 16 & 10 & 12 & 13 & 10 \\
\hline Num. Orden & 1 & 2 & 3 & 4 & 5 & 6 & 7 & 8 & 9 & 10 \\
\hline \multicolumn{11}{|c|}{ Caract. asociación y unidades superiores: } \\
\hline Eryngium dilatatum & $3-3$ & $4-4$ & $3-3$ & $1-1$ & $2-2$ & $2-2$ & $2-2$ & $3-2$ & $1-1$ & $2-2$ \\
\hline Jasonia tuberosa & $3-3$ & $4-4$ & $4-4$ & $4-4$ & $4-4$ & $5-5$ & $2-2$ & $4-4$ & $4-4$ & $4-4$ \\
\hline Linum tenue & $1-1$ & $1-1$ & + & $1-1$ & + & $1-1$ & $1-1$ & $1-1$ & - & $2-2$ \\
\hline Agrostis castellana var. mutica & $1-1$ & + & $1-1$ & - & - & - & $1-1$ & $1-2$ & + & + \\
\hline Phleum bertolonii & $1-1$ & $1-1$ & $1-1$ & $1-1$ & $1-1$ & - & - & - & + & $1-1$ \\
\hline Agrostis nebulosa & + & $1-1$ & - & - & - & - & - & + & + & + \\
\hline Carduncellus cuatrecasasii & - & $1-1$ & $1-1$ & $2-2$ & $2-2$ & $1-1$ & $1-1$ & - & - & - \\
\hline Lotus uliginosus & $1-1$ & - & $1-1$ & - & - & - & $1-1$ & - & - & - \\
\hline Achillea ageratum & $1-1$ & $1-1$ & $1-1$ & - & - & - & $1-1$ & - & - & - \\
\hline Stachys heraclea & - & + & - & + & - & + & + & - & - & - \\
\hline Centaurea castellana & $1-1$ & - & $1-1$ & - & - & - & - & - & - & - \\
\hline Elymus repens & $1-1$ & - & $1-1$ & - & - & - & - & - & - & - \\
\hline Holcus lanatus & + & - & - & + & + & - & - & - & - & - \\
\hline Juncus fontanesii & - & + & $1-1$ & - & - & - & - & - & - & - \\
\hline Prunella hyssopifolia & - & $2-2$ & $2-2$ & $1-1$ & $1-1$ & - & - & - & $1-2$ & - \\
\hline Carex mairii & - & - & - & - & - & - & - & $1-1$ & $1-1$ & + \\
\hline Potentilla reptans & - & - & - & - & - & - & - & + & $1-1$ & + \\
\hline \multicolumn{11}{|l|}{ Caract. Festuco-Brometea: } \\
\hline Brachypodium phoenicoides & + & $1-1$ & $1-1$ & - & $1-1$ & $1-1$ & $1-1$ & - & - & + \\
\hline Dactylis glomerata subsp. hispanica & + & $1-1$ & $1-1$ & - & - & - & - & + & $1-1$ & + \\
\hline \multirow{2}{*}{\multicolumn{11}{|c|}{ Caract. Lavandulo-Genistion boissieri: }} \\
\hline & & & & & & & & & & \\
\hline $\begin{array}{l}\text { Bupleurum rigidum } \\
\text { Avenula gervaisii }\end{array}$ & + & + & + & + & - & - & - & - & - & - \\
\hline $\begin{array}{l}\text { Avenula gervaisii } \\
\text { Thymus zygis subsp. gracilis }\end{array}$ & + & $1-1$ & $1-1$ & - & - & - & - & - & - & - \\
\hline $\begin{array}{l}\text { Thymus zygis subsp. gracilis } \\
\text { Lavandula latifolia }\end{array}$ & - & + & - & + & + & + & + & - & - & - \\
\hline Lavandula latifolia & - & + & - & $1-1$ & - & + & - & - & - & - \\
\hline \multicolumn{11}{|l|}{ Caract. Stellarietea mediae: } \\
\hline $\begin{array}{l}\text { Phlomis herba-venti } \\
\text { Trifolium angustifolium }\end{array}$ & + & - & + & - & + & + & - & - & - & - \\
\hline Trifolium angustifolium & + & + & + & $1-1$ & $1-1$ & - & - & + & - & - \\
\hline Gastridium ventricosum & + & - & - & - & $1-1$ & - & - & - & - & - \\
\hline Scabiosa atropurpurea & $1-1$ & + & $1-1$ & $1-1$ & - & - & - & - & - & - \\
\hline Trifolium campestre & + & - & + & $1-1$ & - & - & - & - & - & - \\
\hline Aegilops geniculata & - & - & - & $1-1$ & - & + & + & - & - & - \\
\hline \multicolumn{11}{|l|}{ Otras compañeras: } \\
\hline Cleonia lusitanica & + & + & + & - & $1-1$ & - & - & - & - & - \\
\hline Avenula bromoides & - & - & - & - & + & - & - & + & + & - \\
\hline Arrhenatherum album & - & - & - & - & - & + & + & - & - & - \\
\hline
\end{tabular}

Otros táxones: Juncus bufonius 1-1 en 2; Rosa pouzinii y Crataegus monogyna + en 3; Poa trivialis subsp. trivialis + en 4; Aegilops triuncialis, Agrostis reuteri, Daucus carota subsp. maximus y Convolvulus arvensis + en 5; Linum suffruticosum, Filipendula vulgaris y Sanguisorba minor subsp. minor + en 6; Bupleurum fruticosum + en 7; Crupina vulgaris + en 8; Scirpus holoschoenus y Trifolium stellatum + en 9; Melissa officinalis + en 10 .

Procedencia de los inventarios: 1.- Próx. Cortijo Carboneros (Valdepeñas de Jaén); 2, 3 y 4.- Crtra subida a Puerto Viejo (Los Villares); 5.- Puerto Viejo (Los Villares); 6.- Puerto La Olla (Los Villares); 7.Urbanización La Pandera (Los Villares); 8 y 9.- Collado del Agua Las Penas (Sierra Las Villas, Jaén); 10.Proximidades Pantano Aguascebas (Sierra Las Villas, Jaén). 
hasta el comienzo del periodo estival en el que la comunidad toma un tono azulado-amarillento debido a las especies directrices de la asociación. En el cortejo florístico abundan plantas propias de este tipo de medios como Achillea ageratum, Centaurea castellana y Prunella hyssopifolia, junto a algunas especies de Agrostietalia castellanae, como Agrostis castellana var. mutica. Se trata de una comunidad muy próxima al Sanguisorbo lateriflorae-Deschampsietum hispanicae del que difiere por la ausencia de Deschampsia hispanica, Plantago maritima subsp. serpentina, Leontodon carpetanus y Sanguisorba lateriflora, junto a la contínua presencia de Eryngium dilatatum, Linun tenue y Carduncellus cuatrecasasii, éste último endémico del sur de España.

Presenta una distribución al menos subbética, bajo termotipos mesosupramediterráneo inferior y ombrotipo subhúmedo (Cano et al., 1999). En áreas más térmicas y húmedas es sustituida por las asociaciones de la alianza Gaudinio fragilisHordeion bulbosi Galán, Deil, Haug \& Vicente Orellana 1997 (Galán de Mera et al., 1997; Pérez Latorre et al., 1998).

Desde el punto de vista topográfico ocupa una posición intermedia entre las comunidades de carácter climático y la vegetación netamente edafófila de suelos hidromorfos. Hacia suelos más húmedos contacta con juncales de Holoschoenetalia o con comunidades de Juncus fontanesii, dependiendo de que la humedad sea menor o mayor. Hacia zonas más secas establece contactos con fenalares de Brachypodium phoenicoides o con matorrales basófilos de Lavandulo-Genistion boissieri Rivas Goday \& Rivas Martínez 1969, como queda reflejado en la tabla 2 por la presencia de Avenula gervaisii, Thymus zygis subsp. gracilis, Lavandula latifolia y Linum suffruticosum.

Por ser comunidades edafohigrófilas resultan muy escasas en la zona, ocupan extensiones reducidas y se hallan sometidas normalmente a un intenso pastoreo, lo que queda de manifiesto en algunos inventarios con especies nitrófilas como Phlomis herbaventi, Trifolium angustifolium, Gastridium ventricosum o Aegilops geniculata. Aparece en la tesela de los encinares del Paeonio coriaceae-Querceto rotundifoliae $\mathrm{S}$. en su faciación más húmeda con Quercus faginea.

\section{ESQUEMA SINTAXONÓMICO}

\section{MOLINIO-ARRHENATHERETEA Tüxen 1937 \\ + Ord. Holoschoenetalia Br.-Bl. ex Tchou 1948 \\ * All. Deschampsion mediae Br.-B1. in Br.- B1., Roussine \& Nègre 1952}

Deschampsietum mediae $\mathrm{Br}$.-Bl. in $\mathrm{Br}$.B1., Roussine \& Nègre 1952 [Praderas de gramíneas y herbáceas con encharcamiento temporal y de carácter submediterráneo: Francia mediteránea y prepirineos aragoneses y catalanes]

\section{Sanguisorbo}

lateriflorae-

Deschampsietum hispanicae Rivas Martínez \& G. López in G. López 1978 corr. Rivas Martínez, Fernández González, Sánchez Mata \& Pizarro 1990 (Syn: Deschampsietum mediae Br.-Bl. in Br.Bl., Roussine \& Nègre 1952 sensu Rivas Goday \& Borja 1961) [Praderas de gramíneas y herbáceas con encharcamiento temporal, de matiz más continental: castellanomaestrazgo-manchegas con penetraciones subbéticas]

Agrostio stoloniferae-Achilleetum agerati $\mathrm{Br} .-\mathrm{Bl}$. in $\mathrm{Br}$.-Bl., Roussine \& Nègre 1952 [Praderas de camomilas con encharcamiento temporal y de carácter submediterráneo: Francia mediterránea y prepirineos aragoneses y catalanes]

Plantagini serpentinae-Jasonietum tuberosae O. Bolòs \& Masalles in O. Bolòs 1983 (Syn: Deschampsietum mediae Br.-B1. in Br.-Bl. Roussine \& Nègre 1952 inulo- 
plantaginetosum serpentinae O. Bolòs 1959) [Praderas de herbáceas con encharcamiento temporal, prepirineo catalán]

Prunello hyssopifoliae-Plantaginetum serpentinae Biurrun 1999 [Praderas de gramíneas y herbáceas con encharcamiento temporal, castellano-cantábricas y navarroalavesas]

\section{Eryngio dilatati-Jasonietum tuberosae} Torres \& Cano ass. nov. [ Praderas de herbáceas con encharcamiento temporal, subbético]

\section{BIBLIOGRAFÍA}

AGUilar, J., G. DELGADO, R. DELGADO, M. DELGADO, J. FERNÁNDEZ, R. NOGALES, E. ORTEGA, J. PÁRRAGA, I. SAURA, C. SIERRA Y M. SIMÓN -1987-Memoria del mapa de suelos de la provincia de Jaén. Escala 1:200.000. Departamento de Edafología y Química Agrícola. Universidad de Granada.

BIURRUN, I. -1999- Flora y vegetación de los ríos y humedales de Navarra. Guineana 5: 1-338.

BOLÒS, O. -1959- El paisatge vegetal de dues comarques naturals: al Selva i la Plana de Vic. Institut Estudis Catalans. Arx. Secc. Ciènc. 26. I.E.C., Barcelona.

BOLÒs, O. -1967- Comunidades vegetales de las comarcas próximas al litoral situadas entre los ríos Llobregat y Segura. Mem. Real Acad. Ci. Barcelona 38(1): 1-269.

BOLÒS, O. -1983- La vegetació del Montseny. Servei de Parcs Naturals. Diputació de Barcelona.

BOLÒS, O. -1996- Contribució al coneixement de la vegetació del territori Auso-Segàrric. Mem. Real Acad. Ci. Barcelona 55(4): 147-272.

BRAUN-BLANQUET, J., N. ROUSSINE \& R. NÈGRE -1952-Les Groupements Végéteux de la France Méditerranéenne. Centre National de la Recherche Scientifique. Montpellier.

CANO, E., J.A. TORRES, A. GARCÍA FUENTES, C. SALAZAR, M. MELENDO, L. RUIZ y J. NIETO -1999-Vegetación de la provincia de Jaén: Campiña, Depresión del Guadiana menor y Sierras Subbéticas. Serv. Public. Universidad de Jaén.
CASTROVIEJO, S. \& al. (eds.) 1986-1999. Flora Iberica, C.S.I.C., Madrid.

GALÁN DE MERA, A., U. DEIL, H. HAUG y J.A. VICENTE ORELLANA - 1997- Contribución a la clasificación fitosociológica de los pastizales de la provincia de Cádiz (España). Acta Bot. Malacitana 22: 147-170.

GARCÍA DUEÑAS, V. -1986- Mapa geológico de España E 1:200.000, Jaén (2a edición). I.G.M.E..

GARCÍA MIJANGOS, I. - 1997- Flora y vegetación de los Montes Obarenses (Burgos). Guineana 3: 1-457.

LOIDI, J., I. BIURRUN y M. HERRERA - 1997- La vegetación del centro-septentrional de España. Itinera Geobot. 9: 386-387.

LÓPEZ GONZÁLEZ, G. -1978-Contribución al conocimiento fitosociológico de la serranía de Cuenca II. Comunidades herbáceas: vegetación de rocas y pedreras; comunidades acuáticas; prados húmedos y juncales; praderas y pastizales; malezas ruderales y arvenses. Anales Inst. Bot. Cavanilles 34(2): 571-596.

LÓPEZ VÉLEZ, G. -1996- Flora y vegetación del macizo del Calar del Mundo y sierras adyacentes del sur de Albacete. Inst. Estudios Albacetenses de la Excma. Diputación de Albacete.

MATEO SANZ, G. -1983-Estudio sobre la flora y vegetación de las sierras de Mira y Talayuelas. Monografias ICONA, nº 31, 290 pp.

PÉREZ LATORRE, A.V., P. NAVAS, D. NAVAS, Y. GIL y B. CABEZUDO -1998- Datos sobre la flora y vegetación de la Serranía de Ronda (Málaga, España). Acta Bot. Malacitana 23: 149-191.

RIVAS GODAY, S. y J. BORJA - 1961 - Estudio de la vegetación y flórula del macizo de Gúdar y Javalambre. Anales Inst. Bot. Cavanilles 19: 1550.

RIVAS MARTínEZ, S. -1996- Clasificación bioclimática de la Tierra. Folia Bot. Matritensis 16: 1-33.

RIVAS MARTÍNEZ, S., A. ASENSI, J. MOLERO \& F. VALLE - 1997- Biogeographical synthesis of Andalusia (southern Spain). J. Biogeogr. 24: 915-928.

RIVAS MARTÍNEZ, S., F. FERNÁNDEZ GONZÁLEZ \& J. LOIDI -1999- Checklist of plant communities of Iberian peninsula, Balearic and Canary islands to suballiance level. Itinera 
Geobot. 13: 353-451.

ROMO, A. M. -1989- Flora y Vegetació del Montsec (Pre-pirineus catalans). Institut Estudis Catalans. Arx. Secc. Ciènc 90. I.E.C., Barcelona. TUTIN, T.G. \& al. (eds.) -1964-1980- Flora Europaea, Vol 1-5. Cambridge Univ. Press, Cambridge.

VALDÉS, B., S. TALAVERA y E. FERNÁNDEZ GALIANO (eds) -1987- Flora vascular de Andalucía Occidental. Volúmenes 1, 2 y 3. Ketres Editora, S.A., Barcelona.
VIGO, J. -1996- El poblament vegetal de la vall de Ribes. Institut Cartogràfic de Catalunya.

Aceptado para su publicación en Junio de 2000

Dirección de los autores. Dpto Biología Animal, Vegetal y Ecología, Fac. CC. Experimentales, Universidad de Jaén. Paraje las Lagunillas s/n, E23071-Jaén. E-mail: jatorres@ujaen.es. 